\title{
Cardenolides, induced responses, and interactions between above- and belowground herbivores of milkweed (Asclepias spp.)
}

\author{
Sergio Rasmann, ${ }^{1}$ Anurag A. Agrawal, Susan C. Cook, and Alexis C. Erwin \\ Department of Ecology and Evolutionary Biology, Corson Hall, Cornell University, Ithaca, New York 14853-2701 USA
}

\begin{abstract}
Theory has long predicted allocation patterns for plant defense against herbivory, but only recently have both above- and belowground plant defenses been considered simultaneously. Milkweeds in the genus Asclepias are a classic chemically defended clade of plants with toxic cardenolides (cardiac glycosides) and pressurized latex employed as anti-herbivore weapons. Here we combine a comparative approach to investigate broadscale patterns in allocation to root vs. shoot defenses across species with a species-specific experimental approach to identify the consequences of defense allocational shifts on a specialist herbivore. Our results show phylogenetic conservatism for inducibility of shoot cardenolides by an aboveground herbivore, with only four closely related tropical species showing significant induction; the eight temperate species examined were not inducible. Allocation to root and shoot cardenolides was positively correlated across species, and this relationship was maintained after accounting for phylogenetic nonindependence. In contrast to long-standing theoretical predictions, we found no evidence for a trade-off between constitutive and induced cardenolides; indeed the two were positively correlated across species in both roots and shoots. Finally, specialist root and shoot herbivores of common milkweed (A. syriaca) had opposing effects on latex production, and these effects had consequences for caterpillar growth consistent with latex providing resistance. Although cardenolides were not affected by our treatments, $A$. syriaca allocated $40 \%$ more cardenolides to shoots over roots. We conclude that constitutive and inducible defenses are not trading off across plant species, and shoots of Asclepias are more inducible than roots. Phylogenetic conservatism cannot explain the observed patterns of cardenolide levels across species, but inducibility per se was conserved in a tropical clade. Finally, given that above- and belowground herbivores can systemically alter the defensive phenotype of plants, we concur with recent calls for a wholeplant perspective in testing models of plant defense allocation.
\end{abstract}

Key words: allocation trade-offs; Asclepias syriaca; cardenolides; constitutive vs. inducible resistance; Danaus plexippus; latex; monarch butterfly; plant defense theory; plant-insect interactions; root herbivory; root vs. shoot; Tetraopes tetraophthalmus.

\section{INTRODUCTION}

Plant traits such as secondary metabolites help to resist herbivory and may be expressed constitutively or induced upon attack. Constitutive resistance is expressed independent of injury, whereas induced resistance is activated or enhanced after the plant has been damaged (Karban and Baldwin 1997). Both of these modes of resistance have been shown to be costly in terms of plant fitness (Strauss et al. 2002) and theory has long predicted that constitutive and inducible resistances should trade off (McKey 1974, Rhoades 1979, Brody and Karban 1992, Thaler and Karban 1997, Koricheva et al. 2004, Morris et al. 2006). It has been proposed that species will rely more strongly on a single mode of defense, depending on the risk of herbivory (Zangerl and Bazzaz 1992). For example, inducibility may be unnecessary for

Manuscript received 15 October 2008; revised 16 December 2008; accepted 9 January 2009. Corresponding Editor: N. M. van Dam.

${ }^{1}$ E-mail: sgr37@cornell.edu species that are heavily attacked by herbivores and that invest predominantly in constitutive resistance.

Types of defenses differ not only among species or genotypes, but also between plant organs such as roots and shoots within a single species. Thus, we must take both above- and belowground organs into account to fully understand the allocation patterns of defensive chemicals, as well as the constraints on their expression (Agrawal 2004, Bezemer and van Dam 2005, De Deyn and Van der Putten 2005, Rasmann and Agrawal 2008, van Dam et al. 2009). Zangerl and Rutledge (1996) were the first to meld ideas about evolutionary trade-offs with patterns of defense allocation in plants. They showed that flowers and leaves of wild parsnip (Pastinaca sativa L.) were the most likely to experience herbivory and contained the highest constitutive concentration of a toxic compound (xanthotoxin). Parsnip roots, however, which experienced less predictable herbivory, had low constitutive defenses but highly inducible xanthotoxin compared to the aboveground parts of the plant. 
Recent surveys of root chemical defenses showed no consistent pattern of allocation toward roots vs. shoots across taxa (Kaplan et al. 2008b, Rasmann and Agrawal 2008, van Dam et al. 2009). The concentration of constitutive secondary compounds in roots and shoots depended on plant family, species, and genotype (Collantes et al. 1998, 1999, Agerbirk et al. 2003, Hol et al. 2004), plant age (Hara et al. 2000, Frolich et al. 2006), and ontogenetic stage of the tissues sampled (Ohnmeiss and Baldwin 2000, Walls et al. 2005). Induction of the same secondary compounds also showed no consistent pattern. For example, after above- or belowground herbivory, root and shoot defenses can be up- (Baldwin 1998, Schmelz et al. 1999, Bezemer et al. 2004) or downregulated (van Dam et al. 2004, Rasmann and Turlings 2007).

To address allocation patterns of constitutive and induced chemical defenses in shoots and roots, and potential interactions between above- and belowground herbivores, we have been studying milkweeds (Asclepias spp.) and their well-known cardenolide defense system. Studying defense expression across different species (i.e., taking a comparative approach) should reveal common mechanisms or constraints that have persisted through the macroevolutionary process (Agrawal 2007). A comparative study of allocation patterns of secondary metabolites across Asclepias spp. should also help to disentangle general patterns of defense allocation to roots and shoots. However, a closer examination of the ecological impacts of root and shoot herbivory in specific species can reveal functional and ecological interactions not visible from larger scale patterns. For example, many chemically mediated plant-herbivore interactions are species specific, and may therefore be difficult to predict simply from measures of chemistry (Blau et al. 1978, De Moraes et al. 1998, Hagele and Rowell-Rahier 2000).

In this study we sought to combine a comparative survey with a more experimental approach on a target species. We asked three specific questions. (1) Within a monophyletic clade of 12 milkweeds, how inducible are cardenolides in the shoots and roots of plants damaged aboveground by monarch caterpillars (Danaus plexipus)? (2) Is there a trade-off between constitutive and induced cardenolides, or between allocation to above- vs. belowground defense across the 12 species? (3) In a single species of milkweed (Asclepias syriaca), is the performance of specialist aboveground herbivores (monarch caterpillars) affected by a specialist root feeder (Tetraopes tetraophthalmus), and what plant traits mediate this interaction?

\section{Material AND Methods \\ Natural history}

The milkweeds (Asclepias spp., Apocynaceae) are a monophyletic group distinct from their closest relatives, which belong to the African genus Gomphocarpus (M. Fishbein, personal communication). Asclepias includes about 130 species in North America, including Mesoamerica and the Caribbean, and some 20 species in South America (Woodson 1954, Fishbein et al., in press). The monarch butterfly (Danaus plexippus, Lepidoptera, Danaidae) is a broadly distributed migratory herbivore that feeds on most milkweed species (Ackery and Vane-Wright 1984, Haribal and Renwick 1998, Agrawal and Fishbein 2006). Other insect herbivores, such as the cerambycid beetles in the genus Tetraopes have radiated with milkweeds; each of the 24 Tetraopes species is primarily associated with one or a few milkweed species (Farrell and Mitter 1998, Farrell 2001); see Fig. 1.

Milkweed herbivores have to cope with a powerful arsenal of defenses present in the plant. Probably the most potent of these are cardiac glycosides (cardenolides) and latex (Agrawal 2005, Agrawal and Fishbein 2006). Cardenolides disrupt the sodium and potassium flux in cells, and occur in all milkweed tissues, including latex (Malcolm 1991). Sticky white latex is exuded immediately after milkweed plants are damaged and is delivered via specialized canals (laticifers) to most plant parts, serving as a physical and chemical barrier to the herbivores (Dussourd 1999, Agrawal et al. 2008). However, we did not find any latex exudation after mechanical or insect herbivore damage to the roots of $A$. syriaca and this is discussed later in the paper.

\section{Above- and belowground induced cardenolides in 12 Asclepias species}

To study cardenolide induction in the roots and shoots of milkweeds, we used 12 Asclepias species from the subclade Incarnatae (Woodson 1954), emended by Fishbein et al. (in press) (see Fig. 2). The series Incarnatae is a monophyletic group composed of approximately 15 species from North and Central America, including the Caribbean, and 7 species from South America. We grew eight north-temperate species and four tropical species.

The 12 milkweed species ( $n=114$ plants, $6-12$ plants per species) were germinated at room temperature after stratifying the seeds at $4^{\circ} \mathrm{C}$ on moist filter paper for two weeks. One seedling per pot (10 cm diameter pots) was transplanted in potting soil (Metro-Mix Sun Gro Horticulture Canada CM Ltd., Vancouver, British Columbia, Canada), and grown in a single growth chamber $\left(12 \mathrm{~h}\right.$ daylight, $26^{\circ} \mathrm{C}$ day: $20^{\circ} \mathrm{C}$ night) for six weeks before harvesting. Plants were watered weekly ad libitum and fertilized (N:P:K 21:5:20 150 ppm N [ $\mu \mathrm{g} / \mathrm{g}]$ ) once 10 days after placement in the growth chamber. After 20 days in the growth chamber, approximately half of the plants ( $n=58,3-6$ per species) were exposed to first-instar monarch caterpillars that had been reared in the lab. Plants received between one and three caterpillars, depending on plant size. Although we recognize that this variation in the number of herbivores on plants is suboptimal, we were able to remove all herbivores from plants after 5 days, thereby reducing 


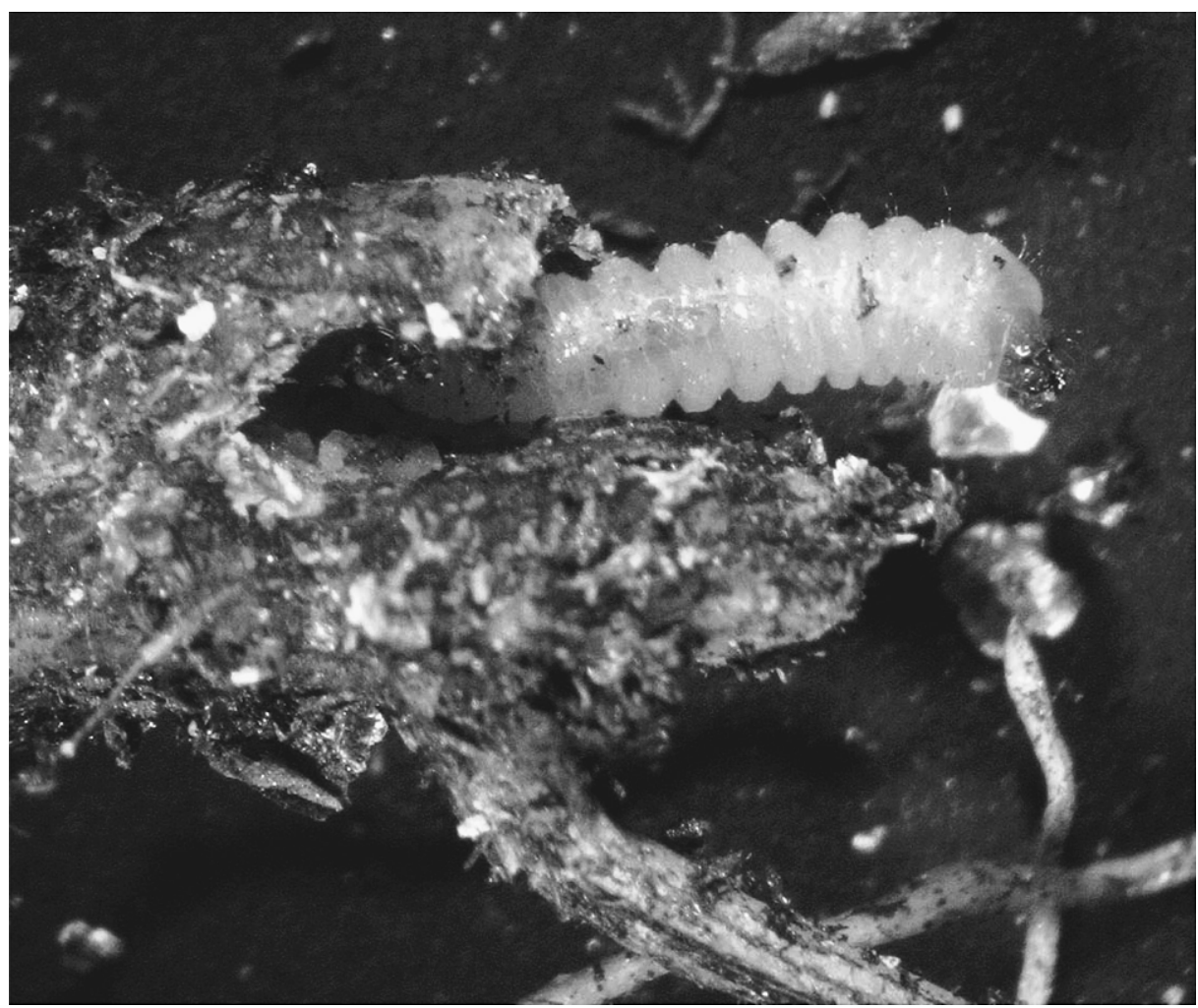

FIg. 1. Larva of the root-specialist longhorn milkweed beetle Tetraopes tetraophthalmus (Coleoptera: Cerambicidae) boring through roots of milkweed, Asclepias syriaca. Photo credit: S. Rasmann.

any bias due to variation in the amount of time needed to impose a fixed proportion (25\%) of leaf damage. The other plants $(n=56,3-7$ per species $)$ remained undamaged. Ten days after cessation of the herbivory treatment, all plants were harvested.

Above- and belowground tissues were separated, roots were washed carefully with water, and all plant material was frozen overnight before being dried fully in a drying oven at $40^{\circ} \mathrm{C}$. We measured cardenolide concentrations with a spectrophotometric assay developed by Brower et al. (1972) and Nelson (1993), adapted to enable the use of a microplate reader. Roots $(n=129$, 2-6 plants/species) were ground using a MM300 Retsch grinder (Retsch GmbH, Haan, Germany) in 10-mL steel grinding vessels at $27 \mathrm{~Hz}$ for $2 \mathrm{~min}$. We weighed $50 \mathrm{mg}$ of dry root tissue into $2 \mathrm{~mL}$ boil-proof microcentrifuge tubes and added $1.9 \mathrm{~mL}$ of $95 \%$ ethanol. Tubes were vortexed, floated in a sonicating water bath $\left(65^{\circ} \mathrm{C}\right)$ for $10 \mathrm{~min}$, and centrifuged at $5000 \mathrm{rpm}$ for $5 \mathrm{~min}$ at room temperature. Two $45-\mu \mathrm{L}$ aliquots of the supernatant from each tube were pipetted into the wells (active sample and blank) of a 96-well plate. We then added 90 $\mu \mathrm{L}$ of ethanol to the blanks and $90 \mu \mathrm{L}$ of $0.15 \%$ 2,204,40-tetranitrodiphenyl (TNDP) in ethanol to the active samples. Finally, $70 \mu \mathrm{L}$ of $0.1 \mathrm{~mol} / \mathrm{L}$ aqueous $\mathrm{NaOH}$ was added to all wells to make the solutions basic and to catalyze the colorimetric reaction. After 18 minutes, all wells in the plate were read at $620 \mathrm{~nm}$ using a microplate reader. On each plate, we devoted six wells to known concentrations of digitoxin (Sigma, CAS No. 71-63-6) in order to generate a standard curve and convert absorbance values to concentrations of cardenolides. We then employed one-way ANOVA to compare the relative allocation to root cardenolides among the species.

To validate our spectrophotometric method to measure cardenolides, we simultaneously analyzed cardenolides from a subset of five Asclepias species (three samples from each species) with the above methods, and by injecting the same samples (after drying and dissolving in methanol), with digitoxin as the internal standard, in a HPLC, and characterized at $218 \mathrm{~nm}$. The two methods were strongly correlated $(n=$ $15, r=0.82, P<0.001$ )

To assess variation in the induced responses of the 12 species, we employed a factorial three-way ANOVA with species, herbivory treatment, and plant part as the main effects. ANOVAs were then performed separately on root and shoot tissues for simplicity of interpretation. We then assessed allocation of cardenolides to roots and shoots by performing Pearson correlations between species means for cardenolides in the absence and presence of herbivory. To gain insight into the macroevolution of root and shoot defense, we estimated 


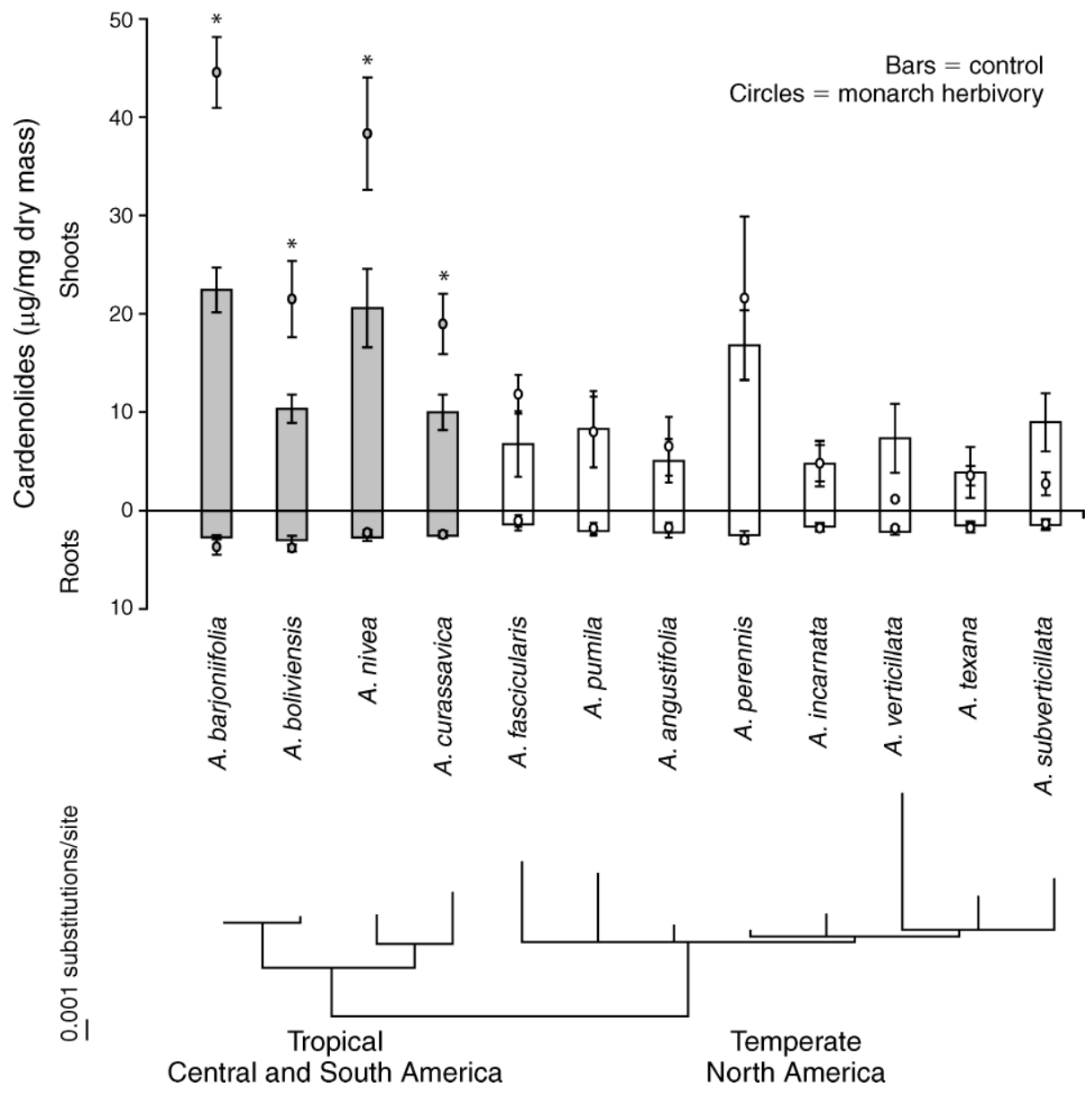

FIG. 2. Root and shoot cardenolide concentrations (mean $\pm \mathrm{SE}$ ) of 12 Asclepias species (series Incarnatae) in undamaged control plants (bars) and plants damaged by monarch caterpillars (circles); $n=2-7$ plants/species. Asterisks above circles indicate significant increases in shoot cardenolide concentrations after induction $(P<0.05)$. Below the data is the corresponding phylogram for the 12 species pruned from a comprehensive phylogeny of Asclepias (Agrawal and Fishbein 2008); the left-hand vertical scale indicates base pair substitutions. Tropical species are represented with solid bars and circles, and temperate species with open bars and circles.

the extent of phylogenetic conservatism (i.e., trait similarity due to shared ancestry) of induced and constitutive cardenolides. Phylogenetic relationships were assessed using a pruned phylogeny of Asclepias, which was the $50 \%$ majority rule consensus of trees sampled from the stationary distribution of a Bayesian analysis of three non-coding cpDNA regions sampled from 145 taxa (Agrawal and Fishbein 2008).

To assess phylogenetic conservatism, we used Pagel's $\lambda$, estimated in BayesTraits (Pagel 1999, 2007, Freckleton et al. 2002, Pagel and Meade 2004, 2007). This parameter is estimated using maximum likelihood (ML) and a generalized least squares (GLS) framework; this value is compared statistically to models where it is set to either zero or 1. A lambda value of 1 indicates phylogenetic conservatism consistent with the tree topology and a random walk model (i.e., trait similarity is directly proportional to the extent of shared evolutionary history). A lambda value of 0 indicates no influence of shared ancestry on trait values (i.e., phylogenetic independence). Values slightly above 1 are possible and indicate that the phenotypes of closely related species are more similar than is predicted from their evolutionary divergence (M. Pagel, personal communication). Models are compared using a likelihood ratio (LR) test, where $\mathrm{LR}=2[\log$-likelihood of the better fitting model - log-likelihood of the worse fitting model]. Here the LR statistic should be $\chi^{2}$ distributed with one degree of freedom (as long as a single parameter is altered between the two models) (Pagel 2007). In addition, phylogenetically independent correlations were performed on these same data using the Continuous module in BayesTraits (Pagel 2007, Pagel and Meade 2007).

To assess negative correlations (trade-offs) in constitutive vs. induced allocation to shoot cardenolides among the 12 species, we employed the test outlined by Morris et al. (2006). This statistical approach 
accounts for several issues that have apparently confounded previous attempts to assess a trade-off between constitutive and induced resistance (Morris et al. 2006). Specifically, this approach uses the difference in mean resistance between damaged and control plants as an optimal metric for induced resistance measurements, and uses a modified Monte Carlo procedure that takes into account sampling variation due to limited sample size, measurement error from environmental and genetic differences, and induced susceptibility, i.e., lower resistance in damaged than in undamaged plants (Morris et al. 2006). The correlation between cardenolide allocation to above- and belowground tissues was also assessed.

\section{Induced resistance above- and belowground} in A. syriaca

Here we used the common milkweed (Asclepias syriaca) and two specialist herbivores, monarch butterfly caterpillars (Danaus plexippus) and larvae of the longhorn milkweed beetle (Tetraopes tetraophthalmus; Coleoptera, Cerambicidae), to address more mechanistically the root-shoot interactions mediated by herbivores and plant defense. The common milkweed is a long-lived, native weedy plant that occurs throughout eastern North America in open habitats such as roadsides, pastures, and old fields. It is the major food source for migrating monarch butterflies (Seiber et al. 1986, Malcolm et al. 1989) and the specialist longhorn beetle $T$. tetraophthalmus, whose adults feed on leaves and flowers and whose larvae feed on milkweed roots (Agrawal 2004).

For these experiments, all insects and seeds were from common milkweed patches naturally found around Ithaca, New York, USA. T. tetraophthalmus adults were kept in large plastic tubs in the laboratory, fed fresh milkweed leaves daily, and given dried grass stems as oviposition sites; see Agrawal (2004). The oviposition substrate was removed from the rearing cages every two days and incubated in the dark at $30^{\circ} \mathrm{C}$ for $7-10$ days. Newly hatched larvae were kept without food in large petri dishes $(10 \mathrm{~cm}$ diameter) on moist filter paper for up to $24 \mathrm{~h}$ before they were placed on the experimental plants. Adult monarchs (10-15 individuals) were kept as a rearing colony in $50 \times 50 \times 50 \mathrm{~cm}$ cages and provided butterfly nectar daily (The Birding Company, Yarmouth, Maine, USA). Females were provided with potted milkweed (A. syriaca) plants for oviposition. Eggs were removed with a paint brush, hatched, and reared on fresh leaves overnight before larvae were placed on the experimental plants. A. syriaca seeds were germinated, planted in $10 \mathrm{~cm}$ diameter pots and grown for 6 weeks in a growth chamber $(12: 12 \mathrm{~h}$ light: dark $26: 20^{\circ} \mathrm{C}$ day: night). Two weeks before the experiment, 84 plants were transplanted to bigger pots $(20 \mathrm{~cm}$ diameter) and randomized in the growth chamber.

To test how belowground herbivory affects the development of monarchs and the defensive traits in
A. syriaca, a fully factorial experiment with four treatments was established ( $n=15$ plants/treatment): one D. plexippus caterpillar (treatment "Monarch"), one D. plexippus and four T. tetraophthalmus larvae (treatment "Monarch and Tetraopes"), four T. tetraophthalmus (treatment "Tetraopes"), and no herbivores (control). D. plexippus caterpillars were placed on the leaves of the plants and T. tetraophthalmus larva were placed $\sim 1 \mathrm{~cm}$ deep into the soil, near the emerging plant stem. Insects were allowed to feed for seven days, after which, D. plexippus caterpillars were removed and weighed on a microbalance. An additional 12 plants in each of "Monarch" and "Monarch and Tetraopes" treatments were manipulated as before, but kept untouched in the chamber for three additional days before $D$. plexippus caterpillars were harvested and weighed. Because the growth and feeding rates of both monarchs and Tetraopes are exponential, this second sampling date was employed to uncover a possible delayed effect of the belowground herbivory on monarchs.

Two plant traits, latex exudation and cardenolide concentration, were sampled at the seven-day harvest. Latex was collected by punching a $6 \mathrm{~mm}$ diameter hole through the tip of the youngest, fully expanded (and if possible, undamaged) leaf of each plant. The latex was collected until no more exudation was observed (about $10 \mathrm{~s}$ after damage) on a previously weighted $1 \mathrm{~cm}$ diameter piece of filter paper. Cardenolides were measured from $50 \mathrm{mg}$ of dried root and shoot material following the same procedure as previously described. The effect of aboveground herbivory (D. plexippus) and belowground herbivory (T. tetraophthalmus) on latex was analyzed with a factorial two-way ANOVA. For analyses of cardenolides, we employed a four-way model to assess the impact of Tetraopes, monarchs, plant part (roots vs. shoots), and all interactions. Here root and shoot tissues are not completely independent, and thus a fourth term "plant" (nested within the Tetraopes $\times$ Monarch interaction) was included in the model to account for the true number of replicates $(n=56)$.

\section{Results \\ Induced cardenolides above and below ground in 12 Asclepias species}

Overall, shoots showed fivefold higher cardenolide concentrations compared to roots (Fig. 2). In the absence of herbivory, the 12 species showed fivefold variation in shoot cardenolides. In the presence of monarch damage, however, there was nearly 13 -fold variation in shoot cardenolides among the same 12 species (Appendix A: Table A1). Across species, monarch herbivory increased shoot cardenolides by $46 \%$, but there was substantial variation among Asclepias species (Appendix A). Roots showed more modest variation in cardenolides, with $2-3$ fold variation across the species in the presence or absence of monarch herbivory. Despite apparent species-specific trends for 


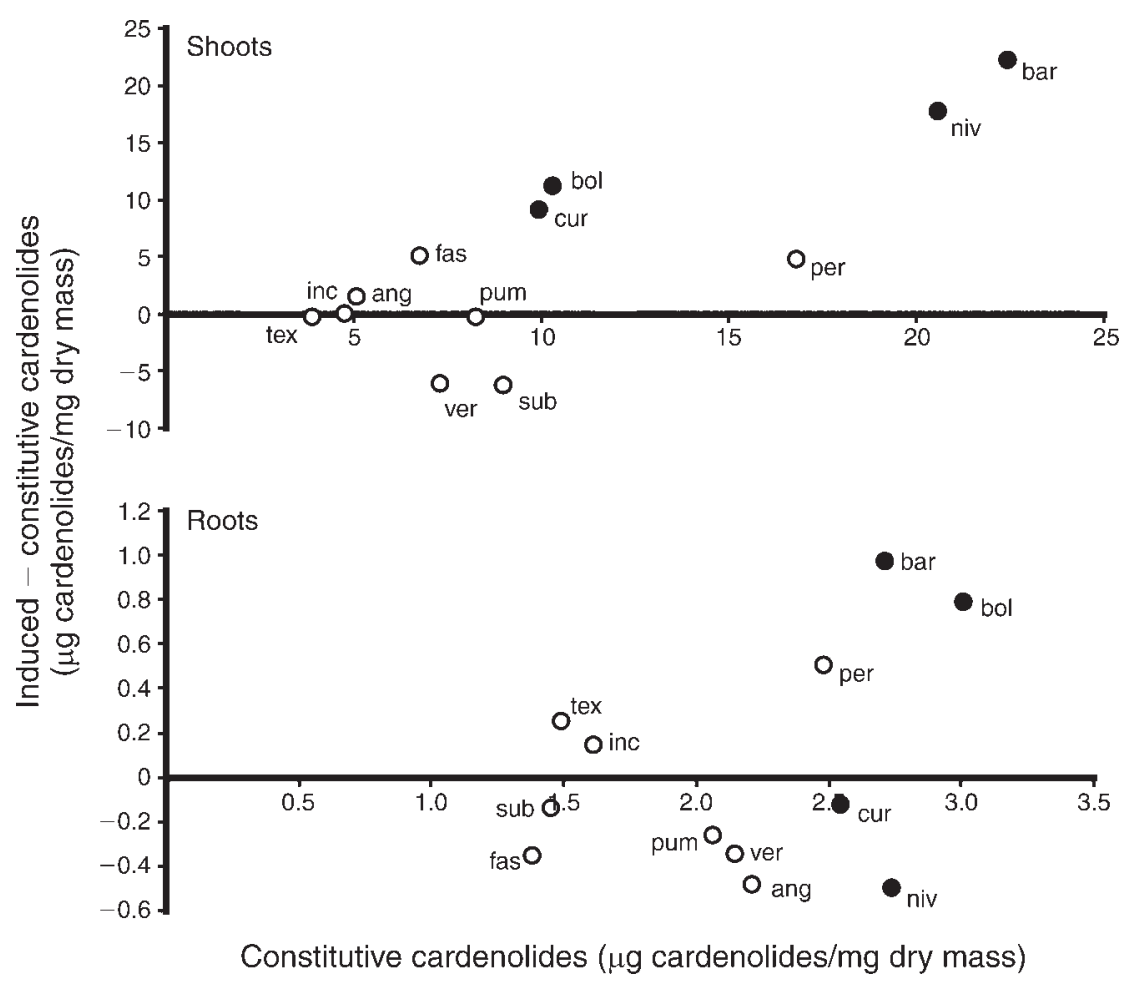

FIG. 3. Raw data for mean investment of constitutive vs. induced (control minus herbivory) cardenolides in shoots and roots for the 12 species of Asclepias in the Incarnatae series. Black circles represent species of the tropical clade; open circles represent species of the temperate zone. Three-letter codes refer to the first three letters of species names (see Fig. 2). Both relationships (roots and shoots) had statistically significant regression slopes (after statistical corrections, see Results: Induced cardenolides above- and belowground in 12 Asclepias species).

up- and down-regulation of cardenolides after shoot herbivory, neither the treatment nor the interaction term was significant for effects on root cardenolides (Appendix A: Table A1).

We found a correlation between above- and belowground cardenolide concentrations, both without $(n=$ $12, r^{2}=0.45, P<0.017$; independent contrast $\lambda=0.206$, $\mathrm{LR}=4.305$ [CI 4.3-4.317], $P=0.038)$ and with aboveground herbivory $\left(n=12, r^{2}=0.51, P<0.009\right.$; independent contrast $\lambda=0.393, \mathrm{LR}=4.357$ [CI 4.3514.63], $P=0.037$ ), showing a concerted investment of cardenolide production above and below ground. We found the same result in a survey of 34 milkweed species (Appendix B).

We next used the phylogeny of this group to map similarities in above- and belowground cardenolides of the 12 species (Figs. 2 and 3). For neither root nor shoot cardenolides were we able to estimate a level of phylogenetic conservatism $(\lambda)$ different from 0 or 1 . Our maximum likelihood estimate of $\lambda$ was 0.188 for shoot cardenolides and 0.566 for root cardenolides (all $P$ s $>0.1$ for differences between the estimated $\lambda$ and set values of 0 or 1 , based on the likelihood ratio test). Quite remarkably, however, our measure of shoot-induced response to monarch caterpillars showed a substantial level of phylogenetic conservatism. All four representatives of the tropical clade showed significant induced cardenolides, whereas none of the north-temperate species showed significant induction: $\lambda=0.648$ (Figs. 2 and 3). This value was different from $0(\mathrm{LH}=6.44$ [CI 6.433-6.448], $P=0.011)$ and from $1(\mathrm{LR}=12.004$ [CI $12.0-12.013], P<0.001)$.

Induction was apparently strongest in the species with the highest constitutive levels (the tropical clade; Fig. 3). The exception here is A. perennis, which showed high levels of constitutive investment in cardenolides, and no induction (Fig. 3).

To formally test for a relationship between constitutive and induced cardenolides, we ran the Monte Carlo procedure developed by Morris et al. (2006). For shoot data, we found strong evidence for a positive association (the opposite of a trade-off) between constitutive and induced allocation to cardenolides. The observed correlation between induced (damage minus control) and constitutive (control) cardenolides was high $(r=$ 0.792 , lower 5 th percentile of the bootstrap distribution of the correlation coefficient $=-0.7413$ ), and the observed slope of the relationship between damaged vs. control species means was positive $(2.127$, lower 5 th percentile $=-0.1501$ ). In only $0.33 \%$ of the randomized data sets did we find a correlation greater than that observed; thus the probability of seeing a correlation as large (or larger) if there was no association between constitutive and induced resistance is 0.0033 . 


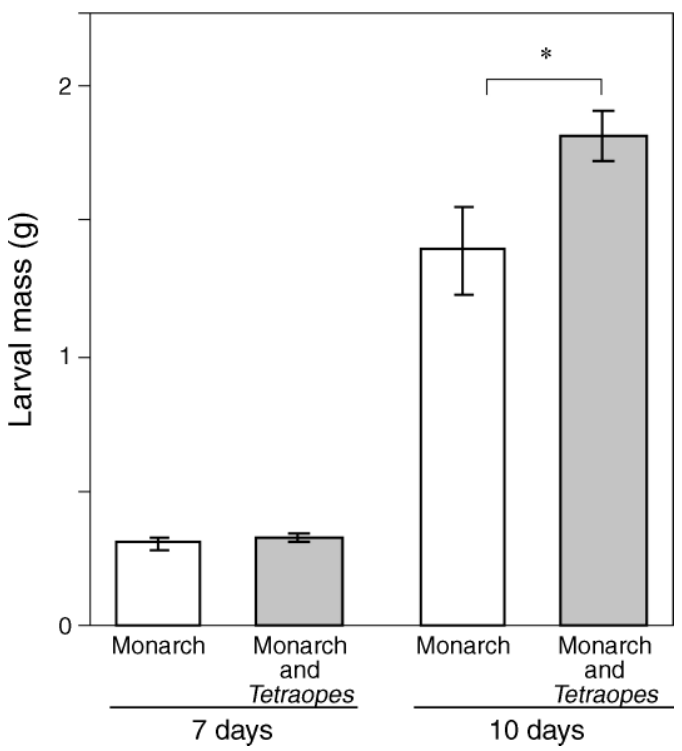

FIG. 4. Mass of monarch caterpillars (mean \pm SE) after seven and 10 days feeding. The asterisk indicates a significant difference $(P<0.05)$ in the 10 -day trial.

The same pattern was found for root data: the positive correlation between induced and constitutive cardenolides $(r=0.377$, lower 5 th percentile of the bootstrap distribution of the correlation coefficient $=$ $-0.781)$ and slope $(1.33$, lower 5 th percentile $=-0.078)$. The probability of finding a correlation as large or larger by chance is 0.014 . We note that this positive correlation between root constitutive and induced cardenolides exists even though we did not detect a significant effect of aboveground herbivory on root chemistry (i.e., this indicates that there was enough variation to estimate induction, and this correlated with the constitutive state).

\section{Resistance in above- and belowground attacked $\mathrm{A}$. syriaca}

After 7 days, monarch larvae feeding on plants alone or with Tetraopes larvae attacking the roots had similar masses $\left(F_{1,23}=0.612, P=0.442\right)$ (Fig. 4). After 10 days of feeding, however, monarchs were $30 \%$ heavier on plants attacked simultaneously by the root herbivore compared to caterpillars feeding on plants without Tetraopes $\left(F_{1,13}=5.324, P=0.038\right)$.

In contrast, we found that latex exudation in the leaves was more than doubled by the feeding of monarchs when compared to the control treatment, but that the effect of monarch herbivory was completely attenuated when plants were attacked belowground by Tetraopes larvae (Fig. 5A, two-way ANOVA, monarch $F_{1,56}=4.683, P=0.035$; Tetraopes $F_{1,56}=1.172, P=$ 0.284 ; interaction: $F_{1,56}=4.942, P=0.030$ ).

Cardenolide concentrations were unaffected by our treatments in the overall analysis, but shoots had 27\% more cardenolides than roots (Fig. 5B; two-way ANOVA: treatment, $F_{3,104}=0.719, P=0.543$; plant part, $F_{1,104}=23.895, P<0.0001$; interaction, $F_{3,104}=$ $0.693, P=0.558)$. Moreover, in analyses of root-shoot cardenolide allocation separated by the four treatments, we found differences between the roots and shoots only in treatments with monarch damage (Fig. 5B; paired $t$ tests: treatment Monarch, difference $=-5.254, t_{14}<$ 0.0001; Monarch and Tetraopes, difference $=-3.52, t_{14}=$ 0.004 ; Tetraopes, difference $=-1.729, t_{15}=0.106$; control, difference $=-1.354, t_{13}=0.2$ ). We cautiously interpret this result as monarch feeding increasing allocation of cardenolides from roots to shoots.

\section{Discussion}

Phylogenetic analyses of plant defense complement more mechanistic studies of within-species variation and selection (Becerra 1997, Wink 2003, Pelser et al. 2005, Agrawal 2007). Here we took a two-sided approach, starting with a comparative survey and concluding with experiments on Asclepias syriaca attacked by specialist herbivores above and below ground. Our results suggest phylogenetic conservatism for inducibility of shoot
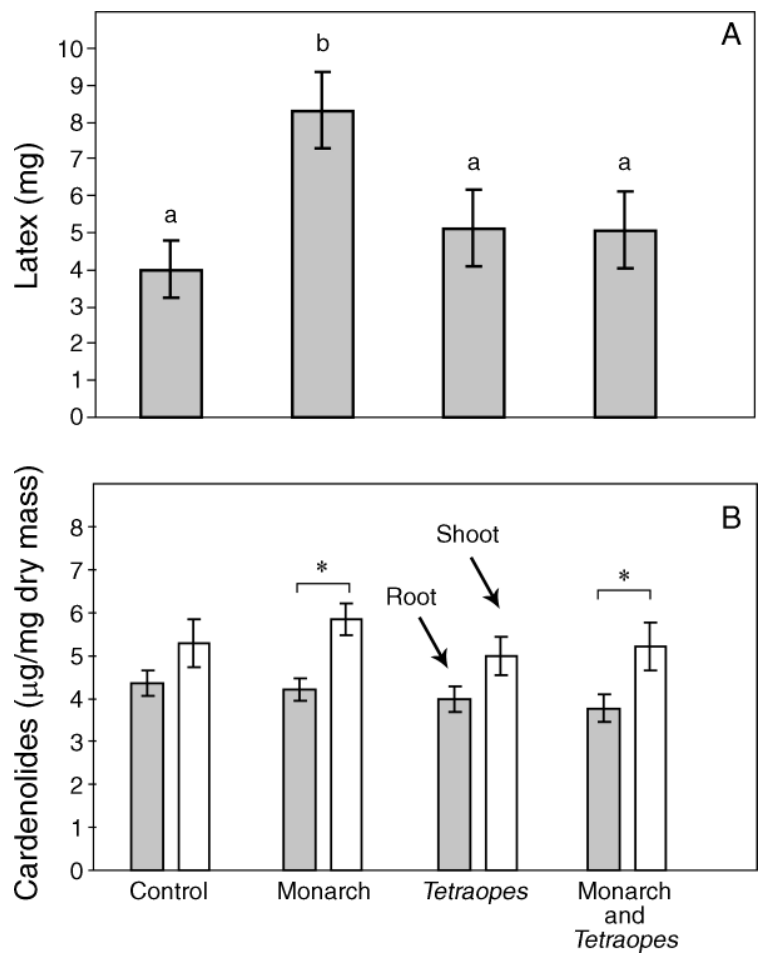

FIG. 5. Defenses in Asclepias syriaca: (A) latex exudation from leaves and (B) cardenolide concentration in roots (gray bars) and shoots (open bars); values are means \pm SE. Latex was collected by punching a $6 \mathrm{~mm}$ diameter hole through the tip of the youngest, fully expanded (and if possible, undamaged) leaf of each plant and collecting until no more exudation was observed. Different letters above bars in (A) represent significant differences $(P<0.05)$, and asterisks in $(\mathrm{B})$ represent significant differences $(P<0.05)$ between root and shoot cardenolides from paired $t$ tests. 
cardenolides. In contrast to long-standing theoretical predictions, we found no evidence for a trade-off between constitutive and induced cardenolides; indeed allocation to the two was strongly positively correlated in both shoots and roots. In addition, allocation to above- and belowground organs was concerted, and these values were positively correlated across species in phylogenetically independent contrasts from two different experiments. Finally, cardenolides were preferentially allocated to shoots over roots in A. syriaca, and both root and shoot herbivores affected latex production. These effects had consequences for monarch caterpillar growth.

\section{Evolution of cardenolide defense in roots and shoots}

Our estimates of phylogenetic conservatism for the Incarnatae clade of Asclepias (12 species) were not distinguishable from either 1 or 0 (in both roots and shoots). In larger data sets we have found some evidence for phylogenetic conservatism in root and shoot cardenolides (Appendix B; Agrawal et al. 2009). Nonetheless, this analysis of 12 species revealed a substantial level of conservatism for shoot inducibility. The interpretation of this result $(\lambda=0.648$, significantly different than both 0 and 1) is that differences in inducibility between species are phylogenetically constrained, but show less similarity among relatives than would be predicted from their genetic divergence (Freckleton et al. 2002). Remarkably, these patterns of inducibility follow a pattern of both phylogenetic and biogeographic constraint: only the tropical species showed significant levels of induction (Figs. 2 and 3).

A recent literature survey of belowground constitutive defenses showed a full panoply of chemicals present in roots that are typically measured in leaves (Kaplan et al. 2008a, Rasmann and Agrawal 2008). However, there was no consistent pattern of greater or lesser allocation of defenses to roots compared to shoots across taxa (e.g., Zangerl and Rutledge 1996, Adler and Wink 2001). Our results with Asclepias showed correlated evolution (i.e., convergence) in allocation to roots and shoots; species that allocate to one organ proportionally allocate to the other. Nonetheless, we found in our analysis of the 12 Incarnatae species and $A$. syriaca that shoots had consistently higher levels of cardenolides than roots.

Both genotype and the environment can modify the levels of defense in roots and shoots. For example, different plant genotypes receiving the same treatment can show different patterns of chemical induction. Root and shoot pyrrolizidine alkaloids in Senecio jacobea were genotypically variable following root or shoot herbivory (Hol et al. 2004). The same genotype induced by different treatments (herbivory, mechanical damage, or phytohormones) also can show different patterns of induction, as was demonstrated for glucosinolates in Brassica spp. (van Dam et al. 2004, van Dam and Raaijmakers 2006), and phytoecdysteroids in Spinacia oleracea (Schmelz et al. 1999, 2002). Here we have shown that aboveground herbivory can enhance latex exudation in A. syriaca, yet the presence of a belowground herbivore can attenuate the induction of latex exudation down to control levels. This result agrees with previous work on latex induction that showed specificity in the strength of the response to different species of herbivore damaging the plant (Van Zandt and Agrawal 2004).

\section{Trade-offs between constitutive and induced responses}

It is generally assumed that anti-herbivore defenses are costly for plants (Karban and Myers 1989, Zangerl and Bazzaz 1992, Karban and Baldwin 1997). For example, it has long been predicted that constitutive and induced defenses should trade off (Koricheva et al. 2004). Recently, however, Morris et al. (2006), have argued that tests of this hypothesis have been statistically flawed because traditional methods to detect tradeoffs have not accounted for limited sample size, errors in measuring resistance, and issues of induced susceptibility (i.e., a decrease in resistance traits after damage). In our analyses employing the methods of Morris et al. (2006), we found no evidence for a trade-off; on the contrary we observed positive correlations between constitutive and induced cardenolides in the shoots and roots of Asclepias. However, we note that this result was driven partly by phylogenetic nonindependence (i.e., four closely related tropical species showed high constitutive cardenolides and high inducibility) (Fig. 4). At the current juncture, we cannot combine phylogenetically independent analyses with the statistically correct tests of trade-offs. However, all other correlations examined in this study were robust to phylogenetic correction, and it appears that there has been positive correlated evolution of root and shoot cardenolide allocation and constitutive and induced responses in Asclepias.

Although there is an apparent general trend for tradeoffs between constitutive and induced resistance from previous analyses (which have not employed the corrections introduced by Morris et al. [2006], reviewed by Koricheva [2004]), our result of a positive association is not unprecedented (e.g., Zangerl and Berenbaum 1990). Only two other studies have taken a comparative approach across taxa to address this question. Thaler and Karban (1997) also reported a positive correlation between constitutive and induced resistance across 21 wild Gossypium (cotton) species, and suggested that constitutive resistance was the ancestral state. Heil et al. (2004), on the other hand, showed the opposite pattern. Facultative myrmecophytic Acacias had highly inducible defenses, but derived species with obligate associations with ants had evolved constitutive traits. Thus, constitutively low species showed strong induction and constitutively high species showed no induction, suggesting a trade-off between these two modes of defense. 
More complex patterns of constitutive and induced resistance have also been suggested. For example, Zangerl and Rutledge (1996) highlighted differential inducibility between organs in wild parsnip (Pastinaca sativa). They showed that flowering parts, which were more likely to be under herbivore attack, contained the highest constitutive concentration of furanocoumarins, while roots, which are exposed to less herbivory, were strongly inducible. Although the relative magnitudes of attack on different plant parts are unknown for Asclepias species, aboveground herbivory and root herbivores could both act as potent agents of selection (Wilbur 1976, Price and Willson 1979, Zalucki and Kitching 1982, Ackery and Vane-Wright 1984, Zalucki et al. 1990, Farrell and Mitter 1998, Farrell 2001, Agrawal 2004, 2005). The highly variable investment in root and shoot defense we saw in our survey data provides testable hypotheses for the probability of attack on these plant organs among the Asclepias species.

\section{Manipulation of resistance traits with above- and belowground herbivory}

We observed that in previously undamaged plants, shoot herbivory increased latex exudation by $48 \%$, but root herbivory reduced it to comparable control levels (Fig. 5). This effect of the root herbivore Tetraopes on latex production was reflected in monarch larval mass, which increased in response to root damage (Fig. 4). Although we did not establish a causal correlation between latex exudation and larval mass in this study, this relationship has been studied in detail, showing that latex exudation reduces larval survival and growth (Dussourd and Eisner 1987, Zalucki et al. 2001, Van Zandt and Agrawal 2004).

Other studies have shown that root herbivory can benefit aboveground herbivores (Gange and Brown 1989, Masters et al. 1993), increase preference for damaged plants (Masters et al. 2001), and even reduce the attractiveness of the plant to predators or parasitoids of herbivores (Rasmann and Turlings 2007). However, the reverse has also been shown: foliar herbivores were affected negatively by enhanced levels of terpenoid aldehydes in the leaves of cotton plants after root herbivory (Bezemer et al. 2002). Agrawal (2004) found that root herbivory in $A$. syriaca plants reduced the abundance of leaf-mining flies by $40 \%$, but only for milkweeds that experienced plant competition.

The specificity of associations between above- and belowground organisms sharing a common resource raises two general predictions. First, the intimate interactions between a herbivore and its host plant seem to be dependent on the presence of other herbivores exploiting the plant. Second, the evolution of plant defenses may involve an intricate combination of above- and belowground selection pressures. Recent work confirms the former prediction (Kaplan et al. 2008a). More work is required to elucidate possible patterns in interactions between above- and below- ground systems that may shape the evolution of plant defense strategies.

Although roots are often similarly (e.g., Wainhouse et al. 1998, Marak et al. 2002, Agerbirk et al. 2003), or even more chemically protected than shoots (e.g., Bezemer et al. 2004, Jorgensen et al. 2005, Walls et al. 2005), we found latex exudation only in the aboveground parts of $A$. syriaca. This is a relatively rare example of strong segregation of defenses. Trichomes and spines may be a similarly segregated form of physical defense. The segregation of latex may result from anatomical or developmental constraints in the production of laticifers (i.e., the canals responsible for latex transportation and exudation), or the fitness consequences for $A$. syriaca of maintaining such traits below ground. Although none of these hypotheses has been investigated, it is clear that belowground herbivory can be substantial for $A$. syriaca, and thus there is potentially strong selection for root defense (Agrawal 2004). Other latex-bearing species (e.g., several Asteraceae and Ipomoea) do contain latex in the roots (Farrell et al. 1991, Snook et al. 1994; personal observations).

Previous work has shown that cardenolides are inducible by $33 \%$ in the aboveground tissues of $A$. syriaca that are attacked by monarch caterpillars or treated with jasmonic acid (Mooney et al. 2008). Thus, it was somewhat surprising that we found no direct evidence for monarch-induced cardenolides in $A$. syriaca in this study, although we did find monarchinducible cardenolides in four other species (Fig. 2). Our work here, both on A. syriaca and 12 other Asclepias species, however, shows no impact of shoot or root herbivory on root cardenolides. Thus, if there are inducible responses to Tetraopes herbivory, they do not appear to be in the form of known direct defenses (i.e., no latex in roots and cardenolides were not affected by root herbivory). We are currently investigating induced indirect defense in milkweed roots; preliminary work shows that entomopathogenic nematodes (Heterorhabditis bacteriophora) are extremely deadly to Tetraopes larvae and are attracted by Tetraopes-infested plants, as previously shown in other systems (Rasmann et al. 2005). Assessing the relative importance of direct defenses (cardenolides) and indirect defense (nematodes) below ground will help to clarify patterns of defense allocation and induction following above- and belowground herbivory.

\section{CONCLuSions}

We speculate that a combination of selection by herbivores, abiotic factors, plant physiological constraints, and phylogentic constraints have lead to the patterns of defense allocation that we see above and below ground in the milkweeds. Less than one-third of the Asclepias species are attacked by specialist Tetraopes, and the determinants of this specificity are currently under investigation. Perhaps some conditions (e.g., wetlands or shaded habitats) are inhospitable for 
root herbivores. Nonetheless, root defenses are not independent from shoot defenses, as the two show positive correlated evolution. Although trade-offs are certainly important in the evolution of defense strategies (e.g., Fine et al. 2004), trade-offs are not universal and concerted patterns of defense allocation appear to be the rule (Agrawal and Fishbein 2006, Agrawal et al. 2008). Finally, phylogenetic conservatism appears to be less constraining in the evolution of defense chemistry itself, but inducibility is more strongly phylogenetically constrained. Future work will assess the role of phytohormonal signaling pathways in the evolution of this induction.

We thus concur with recent calls for the simultaneous study of above- and belowground defense allocation (Van der Putten et al. 2001, van Dam et al. 2003, Bezemer and van Dam 2005, Preisser et al. 2007). Because belowground herbivory is common, and because above- and belowground herbivory result in the manipulation of defense allocation systemically, the time has come for integrating root biology into plant defense theories.

\section{ACKNOWLEDGMENTS}

We thank Mark Fishbein for sharing phylogenetic information and advice on the comparative biology of the milkweeds, Bill Morris for conducting the statistical tests for trade-offs between allocation to constitutive and induced resistance, Kailen Mooney for maintaining the monarch caterpillar colony, and Mike Stastny and anonymous reviewers for comments on the paper. Seeds were provided by Mark Fishbein, Ellen Hornig, Bobby Gendron, Steve Malcolm, Alessandro Rapini, Gary Stell, and Alplains Seeds and Prairie Moon Nursery. This and our other current work is funded by a postdoctoral fellowship from Swiss National Science Foundation PBNEA114692 to S. Rasmann and NSF-DEB 0447550 to A. A. Agrawal.

\section{Literature Cited}

Ackery, P. R., and R. I. Vane-Wright. 1984. Milkweed butterflies: their cladistics and biology. Cornell University Press, Ithaca, New York, USA.

Adler, L. S., and M. Wink. 2001. Transfer of quinolizidine alkaloids from hosts to hemiparasites in two CastillejaLupinus associations: analysis of floral and vegetative tissues. Biochemical Systematics and Ecology 29:551-561.

Agerbirk, N., M. Orgaard, and J. K. Nielsen. 2003. Glucosinolates, flea beetle resistance, and leaf pubescence as taxonomic characters in the genus Barbarea (Brassicaceae) Phytochemistry 63:69-80.

Agrawal, A. A. 2004. Resistance and susceptibility of milkweed: competition, root herbivory, and plant genetic variation. Ecology 85:2118-2133.

Agrawal, A. A. 2005. Natural selection on common milkweed (Asclepias syriaca) by a community of specialized insect herbivores. Evolutionary Ecology Research 7:651-667.

Agrawal, A. A. 2007. Macroevolution of plant defense strategies. Trends in Ecology and Evolution 22:103-109.

Agrawal, A. A., and M. Fishbein. 2006. Plant defense syndromes. Ecology 87(Supplement):S132-S149.

Agrawal, A. A., and M. Fishbein. 2008. Phylogenetic escalation and decline of plant defense strategies. Proceedings of the National Academy of Sciences (USA) 105:10057-10060.

Agrawal, A. A., M. J. Lajeunesse, and M. Fishbein. 2008. Evolution of latex and its constituent defensive chemistry in milkweeds (Asclepias): a phylogenetic test of plant defense escalation. Entomologia Experimentalis et Applicata 128: 126-138.

Agrawal, A. A., J. P. Salminen, and M. Fishbein. 2009. Phylogenetic trends in phenolic metabolism of milkweeds (Asclepias): evidence for escalation. Evolution 63:663-673.

Baldwin, I. T. 1998. Jasmonate-induced responses are costly but benefit plants under attack in native populations. Proceedings of the National Academy of Sciences (USA) 95:81138118.

Becerra, J. X. 1997. Insects on plants: macroevolutionary chemical trends in host use. Science 276:253-256.

Bezemer, T. M., and N. M. van Dam. 2005. Linking aboveground and belowground interactions via induced plant defenses. Trends in Ecology and Evolution 20:617-624.

Bezemer, T. M., R. Wagenaar, N. M. van Dam, W. H. Van der Putten, and F. L. Wackers. 2004. Above- and below-ground terpenoid aldehyde induction in cotton, Gossypium herbaceum, following root and leaf injury. Journal of Chemical Ecology 30:53-67.

Bezemer, T. M., R. Wagenaar, N. M. van Dam, and F. Wäckers. 2002. Interactions between root and shoot feeding insects are mediated by primary and secondary plant compounds. Proceedings of the Section of Experimental and Applied Entomology of the Netherlands Entomological Society (NEV) 13:117-121.

Blau, P. A., P. Feeny, L. Contardo, and D. S. Robson. 1978. Allylglucosinolate and herbivorous caterpillars: contrast in toxicity and tolerance. Science 200:1296-1298.

Brody, A. K., and R. Karban. 1992. Lack of tradeoff between constitutive and induced defenses among varieties of cotton. Oikos 65:301-306.

Brower, L. P., P. B. McEvoy, K. L. Williamson, and M. A. Flannery. 1972. Variation in cardiac glycoside content of monarch butterflies from natural populations in eastern North America. Science 177:426-429.

Collantes, H. G., E. Gianoli, and H. M. Niemeyer. 1998. Changes in growth and chemical defences upon defoliation in maize. Phytochemistry 49:1921-1923.

Collantes, H. G., E. Gianoli, and H. M. Niemeyer. 1999. Defoliation affects chemical defenses in all plant parts of rye seedlings. Journal of Chemical Ecology 25:491-499.

De Deyn, G. B., and W. H. Van der Putten. 2005. Linking aboveground and belowground diversity. Trends in Ecology and Evolution 20:625-633.

De Moraes, C. M., W. J. Lewis, P. W. Paré, H. T. Alborn, and J. H. Tumlinson. 1998. Herbivore-infested plants selectively attract parasitoids. Nature 393:570-573.

Dussourd, D. E. 1999. Behavioral sabotage of plant defense: Do vein cuts and trenches reduce insect exposure to exudate? Journal of Insect Behavior 12:501-515.

Dussourd, D. E., and T. Eisner. 1987. Vein-cutting behavior: insect counterplay to the latex defense of plants. Science 237: 898-901.

Farrell, B. D. 2001. Evolutionary assembly of the milkweed fauna: Cytochrome oxidase I and the age of Tetraopes beetles. Molecular Phylogenetics and Evolution 18:467-478.

Farrell, B. D., D. E. Dussourd, and C. Mitter. 1991. Escalation of plant defense: Do latex and resin canals spur plant diversification? American Naturalist 138:881-900.

Farrell, B. D., and C. Mitter. 1998. The timing of insect/plant diversification: might Tetraopes (Coleoptera: Cerambycidae) and Asclepias (Asclepiadaceae) have co-evolved? Biological Journal of the Linnean Society 63:553-577.

Fine, P. V. A., I. Mesones, and P. D. Coley. 2004. Herbivores promote habitat specialization by trees in Amazonian forests. Science 305:663-665.

Fishbein, M., D. Chuba, C. Ellison, R. Mason-Gamer, and S. P. Lynch. In press. Phylogenetic relationships of Asclepias (Apocynaceae) estimated from non-coding cpDNA sequences. Systematic Botany. 
Freckleton, R. P., P. H. Harvey, and M. Pagel. 2002. Phylogenetic analysis and comparative data: A test and review of evidence. American Naturalist 160:712-726.

Frolich, C., T. Hartmann, and D. Ober. 2006. Tissue distribution and biosynthesis of 1,2-saturated pyrrolizidine alkaloids in Phalaenopsis hybrids (Orchidaceae). Phytochemistry $67: 1493-1502$.

Gange, A. C., and V. K. Brown. 1989. Effects of root herbivory by an insect on a foliar-feeding species, mediated through changes in the host plant. Oecologia 81:38-42.

Hagele, B. F., and M. Rowell-Rahier. 2000. Choice, performance and heritability of performance of specialist and generalist insect herbivores towards cacalol and seneciphylline, two allelochemicals of Adenostyles alpina (Asteraceae). Journal of Evolutionary Biology 13:131-142.

Hara, M., Y. Fujii, Y. Sasada, and T. Kuboi. 2000. cDNA cloning of radish (Raphanus sativus) myrosinase and tissuespecific expression in root. Plant and Cell Physiology 41: $1102-1109$.

Haribal, M., and J. A. A. Renwick. 1998. Identification and distribution of oviposition stimulants for monarch butterflies in hosts and nonhosts. Journal of Chemical Ecology 24: 891-904.

Heil, M., S. Greiner, H. Meimberg, R. Kruger, J. L. Noyer, G. Heubl, K. E. Linsenmair, and W. Boland. 2004. Evolutionary change from induced to constitutive expression of an indirect plant resistance. Nature 430:205-208.

Hol, W. H. G., M. Macel, J. A. van Veen, and E. van der Meijden. 2004. Root damage and aboveground herbivory change concentration and composition of pyrrolizidine alkaloids of Senecio jacobaea. Basic and Applied Ecology 5: 253-260.

Jorgensen, K., S. Bak, P. K. Busk, C. Sorensen, C. E. Olsen, J. Puonti-Kaerlas, and B. L. Moller. 2005. Cassava plants with a depleted cyanogenic glucoside content in leaves and tubers. Distribution of cyanogenic glucosides, their site of synthesis and transport, and blockage of the biosynthesis by RNA interference technology. Plant Physiology 139:363-374.

Kaplan, I., R. Halitschke, A. Kessler, B. J. Rehill, S. Sardanelli, and R. F. Denno. 2008a. Physiological integration of roots and shoots in plant defense strategies links above- and belowground herbivory. Ecology Letters 11:841-851.

Kaplan, I., R. Halitschke, A. Kessler, S. Sardanelli, and R. F. Denno. 2008b. Constitutive and induced defenses to herbivory in above- and belowground plant tissues. Ecology 89: 392-406.

Karban, R., and I. T. Baldwin. 1997. Induced responses to herbivory. First edition. University of Chicago Press, Chicago, Illinois, USA.

Karban, R., and J. H. Myers. 1989. Induced plant responses to herbivory. Annual Review of Ecology and Systematics 20: 331-348.

Koricheva, J., H. Nykanen, and E. Gianoli. 2004. Metaanalysis of trade-offs among plant antiherbivore defenses: Are plants jacks-of-all-trades, masters of all? American Naturalist 163:E64-E75.

Malcolm, S. B. 1991. Cardenolide-mediated interactions between plants and herbivores. Pages 251-296 in G. A. Rosenthal and M. R. Berenbaum, editors. Herbivores: their interactions with secondary metabolites. Academic Press, San Diego, California, USA.

Malcolm, S. B., B. J. Cockrell, and L. P. Brower. 1989. Cardenolide fingerprint of monarch butterflies reared on common milkweed, Asclepias syriaca L. Journal of Chemical Ecology 15:819-853.

Marak, H. B., A. Biere, and J. M. M. Van Damme. 2002. Systemic, genotype-specific induction of two herbivoredeterrent iridoid glycosides in Plantago lanceolata L. in response to fungal infection by Diaporthe adunca (Rob.) niessel. Journal of Chemical Ecology 28:2429-2448.
Masters, G. J., V. K. Brown, and A. C. Gange. 1993. Plant mediated interactions between aboveground and belowground insect herbivores. Oikos 66:148-151.

Masters, G. J., T. H. Jones, and M. Rogers. 2001. Host-plant mediated effects of root herbivory on insect seed predators and their parasitoids. Oecologia 127:246-250.

McKey, D. 1974. Adaptive patterns in alkaloid physiology. American Naturalist 108:305-320.

Mooney, K. A., P. Jones, and A. A. Agrawal. 2008. Coexisting congeners: demography, competition, and interactions with cardenolides for two milkweed-feeding aphids. Oikos 117: $450-458$.

Morris, W. F., M. B. Traw, and J. Bergelson. 2006. On testing for a tradeoff between constitutive and induced resistance. Oikos 112:102-110.

Nelson, C. J. 1993. Sequestration and storage of cardenolides and cardenolide glycosides by Danaus plexippus and $D$. chrysippus petila when reared on Asclepias fruticosa: with a review of some factors that influence sequestration. Pages 95-105 in S. B. Malcolm and M. P. Zaluck, editors. Biology and conservation of the monarch butterfly. Natural History Museum of Los Angeles County, Los Angeles, California, USA.

Ohnmeiss, T. E., and I. T. Baldwin. 2000. Optimal defense theory predicts the ontogeny of an induced nicotine defense. Ecology 81:1765-1783.

Pagel, M. 1999. Inferring the historical patterns of biological evolution. Nature 401:877-884.

Pagel, M. 2007. User's manual for Continuous in BayesTraits. 〈http://www.evolution.rdg.ac.uk/Files/ContinuousManual. pdf $\rangle$

Pagel, M., and A. Meade. 2004. A phylogenetic mixture model for detecting pattern-heterogeneity in gene sequence or character-state data. Systematic Biology 53:571-581.

Pagel, M., and A. Meade. 2007. BayesTraits. 〈http://www. evolution.rdg.ac.uk/Files/BayesTraits-V1.0-Manual.pdf $\rangle$

Pelser, P. B., H. de Vos, C. Theuring, T. Beuerle, K. Vrieling, and T. Hartmann. 2005. Frequent gain and loss of pyrrolizidine alkaloids in the evolution of Senecio section jacobaea (Asteraceae). Phytochemistry 66:1285-1295.

Preisser, E. L., S. E. Gibson, L. S. Adler, and E. E. Lewis. 2007. Underground herbivory and the costs of constitutive defense in tobacco. Acta Oecologica International Journal of Ecology 31:210-215.

Price, P. W., and M. F. Willson. 1979. Abundance of herbivores on six milkweed species in Illinois. American Midland Naturalist 101:76-86.

Rasmann, S., and A. A. Agrawal. 2008. In defense of roots: A research agenda for studying plant resistance to belowground herbivory. Plant Physiology 146:875-880.

Rasmann, S., T. G. Kollner, J. Degenhardt, I. Hiltpold, S. Toepfer, U. Kuhlmann, J. Gershenzon, and T. C. J. Turlings. 2005. Recruitment of entomopathogenic nematodes by insect-damaged maize roots. Nature 434:732-737.

Rasmann, S., and T. C. J. Turlings. 2007. Simultaneous feeding by aboveground and belowground herbivores attenuates plant-mediated attraction of their respective natural enemies. Ecology Letters 10:926-936.

Rhoades, D. F. 1979. Evolution of plant chemical defense against herbivores. Pages 3-54 in J. P. Rosenthal and D. H. Janzen, editors. Herbivores: their interaction with secondary plant metabolites. Academic Press, Orlando, Florida, USA.

Schmelz, E. A., R. J. Grebenok, D. W. Galbraith, and W. S. Bowers. 1999. Insect-induced synthesis of phytoecdysteroids in spinach, Spinacia oleracea. Journal of Chemical Ecology 25:1739-1757.

Schmelz, E. A., R. J. Grebenok, T. E. Ohnmeiss, and W. S. Bowers. 2002. Interactions between Spinacia oleracea and Bradysia impatiens: a role for phytoecdysteroids. Archives of Insect Biochemistry and Physiology 51:204-221. 
Seiber, J. N., L. P. Brower, S. M. Lee, M. M. McChesney, H. T. A. Cheung, C. J. Nelson, and T. R. Watson. 1986. Cardenolide connection between overwintering monarch butterflies from Mexico and their larval food plant, Asclepias syriaca. Journal of Chemical Ecology 12:1157-1170.

Snook, M. E., E. S. Data, and S. J. Kays. 1994. Characterization and quantitation of hexadecyl, octadecyl, and eicosyl esters of p-coumaric acid in the vine and root latex of sweet potato [Ipomoea batatas (L.) Lam]. Journal of Agricultural and Food Chemistry 42:2589-2595.

Strauss, S. Y., J. A. Rudgers, J. A. Lau, and R. E. Irwin. 2002. Direct and ecological costs of resistance to herbivory. Trends in Ecology and Evolution 17:278-285.

Thaler, J. S., and R. Karban. 1997. A phylogenetic reconstruction of constitutive and induced resistance in Gossypium. American Naturalist 149:1139-1146.

van Dam, N. M., J. A. Harvey, F. L. Wackers, T. M. Bezemer, W. H. van der Putten, and L. E. M. Vet. 2003. Interactions between aboveground and belowground induced responses against phytophages. Basic and Applied Ecology 4:63-77.

van Dam, N. M., and C. E. Raaijmakers. 2006. Local and systemic induced responses to cabbage root fly larvae (Delia radicum) in Brassica nigra and B. oleracea. Chemoecology 16: $17-24$.

van Dam, N., T. Tytgat, and J. Kirkegaard. 2009. Root and shoot glucosinolates: a comparison of their diversity, function and interactions in natural and managed ecosystems. Phytochemistry Reviews 8:171-186.

van Dam, N. M., L. Witjes, and A. Svatos. 2004. Interactions between aboveground and belowground induction of glucosinolates in two wild Brassica species. New Phytologist 161: 801-810.

Van der Putten, W. H., L. E. M. Vet, J. A. Harvey, and F. L. Wackers. 2001. Linking above- and belowground multitrophic interactions of plants, herbivores, pathogens, and their antagonists. Trends in Ecology and Evolution 16: $547-554$.

Van Zandt, P. A., and A. A. Agrawal. 2004. Specificity of induced plant responses to specialist herbivores of the common milkweed Asclepias syriaca. Oikos 104:401-409.
Wainhouse, D., R. Ashburner, E. Ward, and J. Rose. 1998. The effect of variation in light and nitrogen on growth and defence in young Sitka Spruce. Functional Ecology 12: 561-572.

Walls, R., H. Appel, M. Cipollini, and J. Schultz. 2005. Fertility, root reserves and the cost of inducible defenses in the perennial plant Solanum carolinense. Journal of Chemical Ecology 31:2263-2288.

Wilbur, H. 1976. Life history evolution in seven milkweeds of the genus Asclepias. Journal of Ecology 64:223-240.

Wink, M. 2003. Evolution of secondary metabolites from an ecological and molecular phylogenetic perspective. Phytochemistry 64:3-19.

Woodson, R. E. 1954. The North American species of Asclepias L. Annals of the Missouri Botanical Garden 41:1-211.

Zalucki, M. P., L. P. Brower, and A. Alonso. 2001. Detrimental effects of latex and cardiac glycosides on survival and growth of first-instar monarch butterfly larvae Danaus plexippus feeding on the sandhill milkweed Asclepias humistrata. Ecological Entomology 26:212-224.

Zalucki, M. P., L. P. Brower, and S. B. Malcolm. 1990. Oviposition by Danaus plexippus in relation to cardenolide content of three Asclepias species in the southeastern USA. Ecological Entomology 15:231-240.

Zalucki, M. P., and R. L. Kitching. 1982. Dynamics of oviposition in Danaus plexippus (Insecta, Lepidoptera) on milkweed, Asclepias spp. Journal of Zoology 198:103-116.

Zangerl, A. R., and F. A. Bazzaz. 1992. Theory and pattern in plant defense allocation. Pages 363-391 in R. S. Fritz and E. L. Simms, editors. Plant resistance to herbivores and pathogens: ecology, evolution and genetics. University of Chicago Press, Chicago, Illinois, USA.

Zangerl, A. R., and M. R. Berenbaum. 1990. Furanocoumarin induction in wild parsnip: genetics and populational variation. Ecology 71:1933-1940.

Zangerl, A. R., and C. E. Rutledge. 1996. The probability of attack and patterns of constitutive and induced defense: a test of optimal defense theory. American Naturalist 147:599-608.

\section{APPENDIX A}

ANOVA table for results of 12 Asclepias species (Ecological Archives E090-169-A1).

\section{APPENDIX B}

Bar graph of root cardenolides in 34 species of Asclepias (Ecological Archives E090-169-A2). 


\section{Ecological Archives E090-169-A1}

Sergio Rasmann, Anurag A. Agrawal, Susan C. Cook, and Alexis C. Erwin. 2009. Cardenolides, induced responses, and interactions between above- and belowground herbivores of milkweed (Asclepias spp.). Ecology 90:2393-2404.

Appendix A. Three-way ANOVAs (and separate and two-way analyses on root and shoot data independently) for effects of species and monarch herbivory on cardenolides of 12 Asclepias species.

\begin{tabular}{|l|l|l|l|r|}
\hline Analysis & \multicolumn{1}{|c|}{ Source } & \multicolumn{1}{c|}{ df } & \multicolumn{1}{c|}{$F$ ratio } & \multicolumn{1}{c|}{$P$ value } \\
\hline Root and shoot & plant part (P) & 1,170 & 216.735 & $<0.0001$ \\
\hline & treatment (T) & 1,170 & 11.363 & 0.0009 \\
\hline & species (S) & 11,170 & 16.641 & $<0.0001$ \\
\hline & $\mathrm{P} \times \mathrm{T}$ & 1,170 & 11.031 & 0.001 \\
\hline & $\mathrm{P} \times \mathrm{S}$ & 11,170 & 13.573 & $<0.0001$ \\
\hline & $\mathrm{T} \times \mathrm{S}$ & 11,170 & 2.939 & 0.001 \\
\hline & $\mathrm{P} \times \mathrm{T} \times \mathrm{S}$ & 11,170 & 2.741 & 0.003 \\
\hline & $\mathrm{T}$ & 1,84 & 0.035 & 0.853 \\
\hline & $\mathrm{S}$ & 11,84 & 4.613 & $<0.0001$ \\
\hline & $\mathrm{T} \times \mathrm{S}$ & 11,84 & 0.54 & 0.871 \\
\hline & $\mathrm{T}$ & 1,84 & 11.579 & 0.001 \\
\hline & $\mathrm{S}$ & 11,84 & 15.573 & $<0.0001$ \\
\hline & $\mathrm{T} \times \mathrm{S}$ & 11,84 & 2.895 & 0.003 \\
\hline
\end{tabular}


Sergio Rasmann, Anurag A. Agrawal, Susan C. Cook, and Alexis C. Erwin. 2009. Cardenolides, induced responses, and interactions between above- and belowground herbivores of milkweed (Asclepias spp.). Ecology 90:2393-2404.

Appendix B. Bar graph of root cardenolides in 34 species of Asclepias.

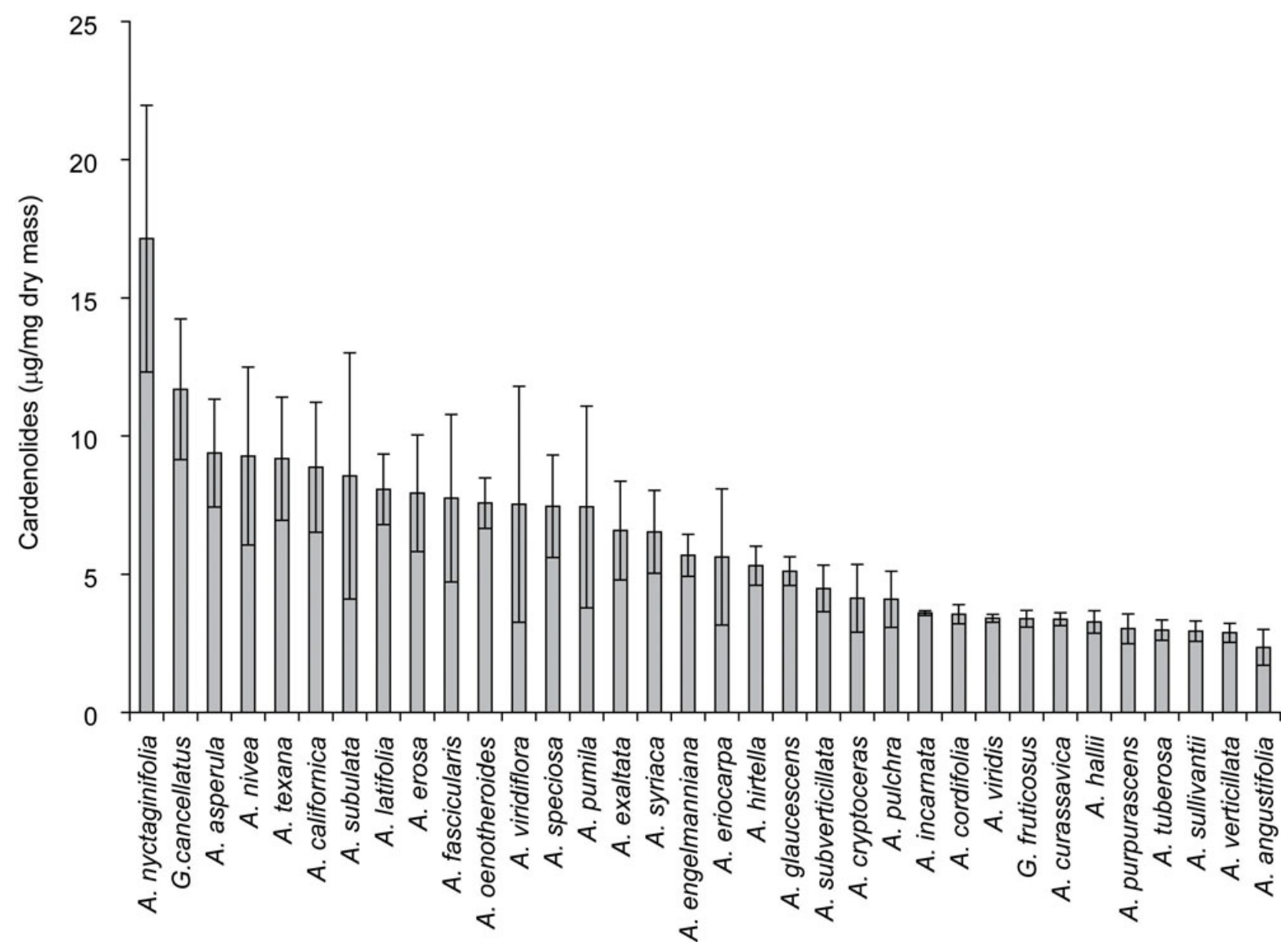

FIG. B1. Cardenolide concentration ( $\mu \mathrm{g} / \mathrm{mg}$ of dry tissue, mean $\pm \mathrm{SE}$ ) in the roots of 32 Asclepias and two Gomphocarpus species. A. pulchra is a subspecies of A. incarnata. $\left(n=2-6\right.$ plants/species, ANOVA, $\left.F_{33,122}=3.35, P<0.0001\right)$. Root cardenolides were compared to a previously published data set of shoot cardenolides from the same set of plants (Agrawal and Fishbein 2008) in order to assess allocation to root and shoot cardenolides. We found that root and shoot cardenolides were positively correlated $(n=34, r=0.348, P=0.044)$, and this was corroborated when using a phylogenetically corrected analysis ( $\lambda=0.709, \mathrm{LH}=10.263$ [CI 10.249-10.286], $P<0.001$, see text for explanation of statistical analysis). 